\title{
Multi-Criteria Model for the Selection of Construction Materials: An Approach Based on Fuzzy Logic
}

\author{
Radojko OBRADOVIĆ*, Dragan PAMUČAR
}

\begin{abstract}
Abstrack: The process of construction and exploitation of a building passes through the preparation phase, the construction phase and the exploitation phase. Each of these phases involves the selection of criteria and decision making, with the aim of selecting the optimal construction materials for the building. The paper presents a new model for selecting construction materials that is based on fuzzy logic. In order to simulate the expert process of assessing the construction and exploitation of a building during which construction materials are selected, a modular fuzzy logic system was designed with a unique base of knowledge to assist in decision making on the priorities of the construction material. The model consists of four modules that are hierarchically organized on two levels. The size of the output from the model is the measure of a material's priority. Each of the materials observed (straw, wood, brick and concrete-steel) is assigned a certain value for the criterion function, on the basis of which the user of the system chooses the material for construction. In addition to the structure of the model, the paper presents its testing. Testing was carried out on the case study of selecting materials for the construction of five residential buildings.
\end{abstract}

Keywords: fuzzy logic; fuzzy logic system; selection of construction materials

\section{INTRODUCTION}

High energy prices and global climate change are leading today's society to change their energy consumer habits. Also, a consequence of inefficient energy consumption is higher production of the same, and thus an unnecessarily greater negative impact on the environment. On the other hand, the rapid development of the housing sector in the 1960s led to the construction of a large number of residential buildings that are today large energy consumers, as they were built at a time when there were no regulations on the energy efficiency of buildings.

In addition, today there are various types of construction materials whose thermo-insulation properties directly affect the energy efficiency of buildings. The process of construction and exploitation of a building can be divided into three phases: the preparation phase, the construction phase and the exploitation phase.

Each of these phases involves selecting criteria and making certain decisions. The significance of a certain phase and how much a certain element influences the final decision depends on the user of the building. The ultimate goal is to choose the optimal material with which the building will be constructed.

In the preparation phase it is important to take into account the site costs, the infrastructure costs, the impact on the environment and the risk of compromising the material. The selection of where to construct a building directly affects the site costs, whereby costs may vary from extremely high in exclusive locations, to extremely low in peripheral urban locations. Another significant element is the cost of infrastructure equipment which, depending on the location, can be very high or extremely low. A cheap location can have distant infrastructure, which can significantly affect the final price. The third factor is the impact on the environment. What kind of effect the building has on the environment depends on the user and his feeling towards the needs and care for the environment and for the place where the building is constructed. The fourth factor in the preparation phase is the possible risk of compromising the material, namely, which material is used for building and how in the preparations for building the material can be compromised.

The construction process is characterized by several important elements: construction speed, the need for a skilled workforce, material availability and construction costs. Construction speed can be of importance to the builder during the time needed to complete the project and the period until moving into the completed building. The need for a skilled workforce affects many factors, from the price to the choice of technology used in the building process. The availability of materials affects the type of construction and the choice of project, while construction costs can be crucial in decision making.

The first two phases usually last a few years, and the exploitation period for buildings is sometimes measured in decades. Some builders place particular importance on the environment around a building. Another important factor is the quality of life in the building, that is, whether the building is constructed of natural materials, whether there are harmful fumes, what the level of protection from mould is, sound insulation, etc. The cost of exploitation is an influential factor that can significantly change the economic viability of a building and drastically increase the costs from the first two phases. The fourth factor is the durability of the building, which is of exceptional importance, since it affects the length of a building's use.

Some of these parameters can be quantified, and some are qualitative in character and directly depend on the subjective preferences of the user of the building under construction. In order to simulate the expert process of assessing the construction and exploitation of a building, during which construction materials are selected, a modular fuzzy logic system was designed with a unique base of knowledge to assist in decision making on preferences for construction materials.

The modular fuzzy logic system consists of four modules, with the first three modules simulating the process of selecting the construction material during the preparation phase, construction phase and the exploitation phase. The fourth module accumulates the decisions made in the previous three phases and as its output it proposes different preferences for construction materials to the user. 
The user chooses the material with the highest degree of preference. Fuzzy sets and fuzzy logic are used to describe the criteria in each of the phases (modules).

Fuzzy sets and fuzzy logic are used because they are a suitable mathematical apparatus for the treatment of uncertainty and indeterminacy. Besides this, they are often successfully applied in support processes for making various decisions. Their place in translating different types of indeterminacy, uncertainty and imprecision into fields of determinacy, certainty and precision is particularly significant, whereby different mathematical operations translate general attitudes into numerical values. In other words, they allow the translation of a completely unstructured set of heuristic assertions expressed in words into an algorithm based on scientific principles [1]. In the following section of the paper the basic fuzzy logic model and fuzzy logic system are presented.

\section{LITERATURE REVIEW}

Generally speaking, all decision-making techniques are designed to shape and formalize a decision-making process most effectively [2]. A large number of multicriteria decision-making (MCDM) techniques have been introduced in the domain of engineering in general, and the construction industry in particular, MCDM techniques are based on different theoretical postulates, using a broad spectrum of data, depending on the expected result. This section offers a systematization of different MCDM techniques that have been used in construction over the past ten years.

Construction is an area that interacts strongly with natural environments. Natural materials found on Earth make a majority of raw materials used in the construction industry, whose processing and treatment, alongside the building process in general, lead to pollution. The paper [2] has explored the Spanish MIVES methodology (English: Integrated Value Model for Sustainable Assessment) defining sustainability criteria for industrial structures and, accordingly, the optimal solution. Broadly speaking, MIVES combines MCDM methods with Multi-Attribute Utility Theory (MAUT), includinga value function concept and weight assignment through AHP modelling [3]. A similar research is presented in [4], where the authors have also used MIVES, but combined with Monte Carlo simulation, to gauge the sustainability of concrete structures. De la Fuente et al [5], too, have exploited MIVES together with the AHP method to reduce a subjective human impact on the selection of materials to be used for sewer pipe installations. Akhtar et al. [6] have addressed the same issue based on the AHP method only. MIVES is used in [7] as well, while assessing the sustainability of alternative types of concrete and reinforcement to be used in tunnel construction, depending on environmental, social and economic criteria. The case study has been realized for the City of Barcelona. Pons and de la Fuente [8] are using MIVES to select best suited concrete pillars as structural components, while Pujadas et al. [9] have employed it to create a framework for heterogeneous public investments, taking a step closer to sustainable urban planning. Various economic, environmental and social aspects have been taken into account, along with five criteria and eight indicators.
The problems surrounding surveillance, repairs and restoration of steel bridge structures pose a serious challenge to engineers, required to make key decisions while at risk of making a very costly mistake. In order to eliminate subjectivity from the selection of alternatives in the given case, Rashidi et al. [10] have presented a Decision Support System (DSS), using a Simplified AHP (S-AHP) method within. The S-AHP has merged the Simple Multi-Attribute Rating Technique (SMART) and the AHP method to assist engineers while making safety, functionality and sustainability provisions for a steel bridge design. Ref. [11] offers a framework for the choice of a bridge construction between the ABC (Accelerated Bridge Construction) method and conventional alternatives, using TOPSIS and Fuzzy TOPSIS methods.

Formisano \& Mazzolani [12] have presented in their paper a new procedure for the selection of an optimal solution for the seismic retrofitting of existing structures, and one for the vertical expansion of an existing permanent structure. The procedure involves the application of three MCDM methods, namely, TOPSIS, Elimination and Choice Expressing Reality (ELECTRE) and Multi-Criteria Optimization and Compromise Solution (VIKOR). In two case studies the methods produced the same results. Terracciano et al. [13] have carried out the selection of cold-formed thin-walled steel structures for vertical reinforcement, and energy retrofitting systems for existing masonry structures. The TOPSIS method has been used for the selection of alternatives, based on structural, economic, environmental and energy criteria.

While upgrading traditional buildings to modern it is necessary to respect technical regulations, energy requirements and demands for comfort, preserving the traditional architecture in the process as well. Šiožinyte et al. [14] use the AHP and Gray-TOPSIS approach to choose an optimal way to modernize traditional buildings.

In [15] the AHP method is used to select an environmentally-friendly method for the construction of a highway, given a strong impact on the environment it might have. The alternatives include different materials, operations and project conditions. The construction of transportation infrastructure can substantially increase the safety of transportation participants and reduce traffic jams. Stević et al. [16] are selecting locations for the construction of roundabouts, using the Rough Best-Worst Method (BWM) and Rough Weighted Aggregated Sum Product Assessment (WASPAS), based on a New Rough Hamy Aggregator.

Rashid et al. [17] have used MCDM methods to select a sustainable concrete mixture combining a conventional coarse aggregate and ceramic waste as an aggregate material. The AHP and TOPSIS methods are used to select the type of concrete performing best in terms of pressures it can sustain and environmental impacts.

During and after the construction of buildings natural resources are used in extreme amounts, which has an adverse effect on the environment. Most of the systems to assess the sustainability of structures involve the environmental aspect only, whereas it is necessary to consider all three basic principles of sustainability. Having this in mind, Raslanas et al. [18] have developed a sustainability assessment system for leasure facilities, using the AHP method. As so-called "green" buildings are 
environmentally friendly, the selection of building materials used in their construction is getting the attention it deserves. Given the complexity of the process, MCDM methods are indispensable. DEMATEL, ANP and ZeroOne Goal Programming (ZOGP) are used in [19].

The selection of a construction project manager is key to the given construction process. Zavadskas et al. [20] and Mardani et al. [21] have used the MCDM approach, employing the AHP and Additive Ratio Assessment (ARAS) methods. Alternatives are selected based on education, experience, personal abilities and skills as criteria.

Following the review of literature presented above, it is clear that multi-criteria decision-making models offer very strong tools to facilitate decision-making in the construction industry. Traditional MCDM techniques imply that the best alternative be selected from a set of alternatives based on a set of evaluation criteria. On the other hand, the traditional multi-criteria techniques do not possess the adaptability necessary to address uncertainties emerging in the environment. This paper offers an original multi-criteria model for the prediction and evaluation of building materials. The model hinges on a fuzzy logic system, where all input parameters are defined by fuzzy sets. One of the advantages of the new model over existing ones is that it provides for a complex analysis of a larger number of parameters affecting the choice of a building material. In this context, a total of 12 criteria, grouped in three clusters, have been analyzed. An additional benefit is that it is processing group knowledge in the process of selecting building materials, as the model contains a unique rule base, arising from an expert knowledge base, built upon the heuristic experience of civil engineers. Another advantage lies in the adaptability of the model, as it allows for a fuzzy rule base to be adjusted, making it possible to manage the system and adapt it to a fuzzy environment.

\section{THE BASIC FUZZY LOGIC MODEL AND FUZZY LOGIC SYSTEM}

Fuzzy logic is most often used to model complex systems in which it is difficult to determine the interdependencies between individual variables using other methods. The application of fuzzy logic in the design of complex systems is very widespread, from designing different models of multi-criteria decision making [1, 23, 24] to automated management systems [25-27].

Models based on fuzzy logic consist of the "If - Then" rules. The "If - Then" rules are interconnected by the expression "Else". An example of the approximate reasoning algorithm is the following set of rules:

If value $X$ is high then value $Y$ is low

Else

If value $X$ is medium then value $Y$ is medium

Else

If value $X$ is low then value $Y$ is high

As we see from these simple rules, the value of output variable $Y$ is conditioned by the value of input variable $X$. Input variable $X$ is called the fuzzy variable. The value of the fuzzy variable is reached by measurement, consideration, and very often subjective assessment based on experience and intuition.
The If part represents the input state, that is, the premise. The Then part is the output state, that is, the consequent part. The conclusion can be in a complex form and then the system has multiple output variables.

A large number of rules in which words describe the solution to a problem represent the base of rules or expert rules. To make it easier to understand the rules, they are written in a suitable order, although the order is essentially irrelevant. The rules are linked by the conjunction $\mathrm{Or}$, which is often not mentioned. Besides the conjunction $\mathrm{Or}$ the rules can also be linked by the conjunction And.

Approximate reasoning is a form of fuzzy logic that contains a set of rules of reasoning whose premises are fuzzy propositions. According to Lotfi Zadeh, approximate reasoning is a form of reasoning that offers a much more natural framework for human reasoning than traditional two-valued logic [22]. In reality, the most common input values are represented by a number, and so the output values are also in numerical form. On the other hand, in a fuzzy system the system is described verbally (qualitatively) by means of production rules. For this reason when using fuzzy logic operations first the numerical values are converted (fuzzified). After this, the mechanism of approximate reasoning processes them in the fuzzy system through the phases of aggregation, activation and accumulation [22, 23]. A numerical output value is obtained by the process of defuzzification. Fig. 1 shows the process of approximate reasoning.

Aggregation is the phase in which the particular values of the membership functions are combined with measured numerical values. It is the process that determines to what degree of confidence (level of truth) a numerical input value belongs to a given fuzzy set. Aggregation is equivalent to fuzzification if there is only one input [22]. This phase is presented in Fig. 1 by a vertical line that crosses the input fuzzy sets. For each set it can be seen how much truth applies to each rule (the shaded part of the triangle).

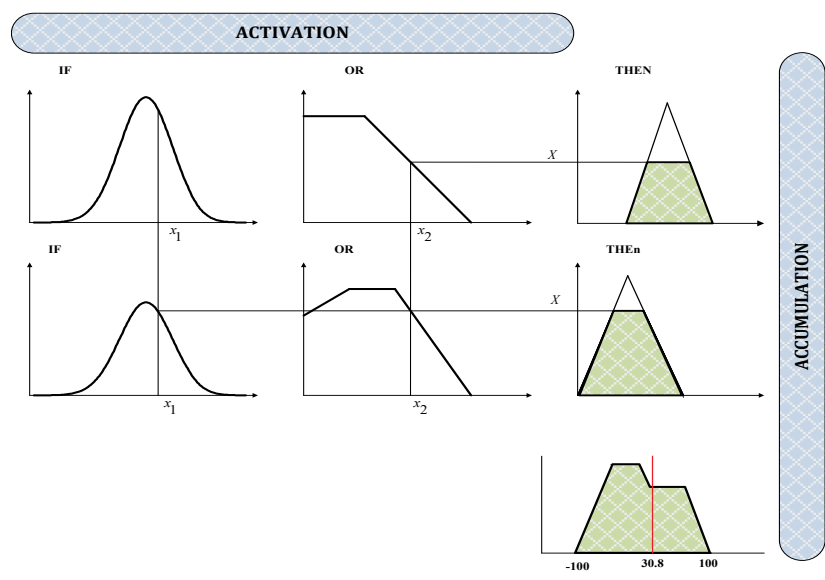

Figure $1 \mathrm{Graphic} \mathrm{presentation} \mathrm{of} \mathrm{the} \mathrm{approximate} \mathrm{reasoning} \mathrm{process}$

If $n$ parallel rules are interpreted using the conjunction Or, Fig. 1, they can be shown using fuzzy relation:

$R=\bigcup_{k=1}^{n} R_{k}$

The membership functions of this relation are shown as: 


$$
\begin{aligned}
\mu_{R}(x, y) & =\underset{k}{\vee} \mu_{R}(x, y)=\max _{k} \mu_{R}(x, y) \\
& \left.=\underset{k}{\max (\min } \mu_{R}(x), \mu_{R}(y)\right)
\end{aligned}
$$

Activation is the deduction of a conclusion reached in the Then section of the rules. In Fig. 2 the shaded part of the triangle shows the activated part of the fuzzy set at the output. Fig. 2 shows the graphic interpretations of two activation methods in the method of direct conclusion (Mamdani method).
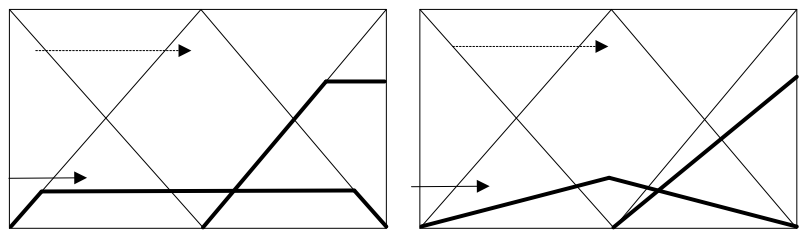

Figure 2Activation phase (MIN - intersecting, PROD - scaling)

As seen in Fig. 2 the MIN method intersects, while the PROD (product) method carries out scaling - proportional reduction.

In the process of accumulation all activated conclusions are accumulated. Accumulation is most often realized by means of two methods: MAX and SUM. Activated conclusions are accumulated in different ways. Fig. 3 shows a graphic interpretation of two methods: MAX and SUM. Fig. 3 on the left shows the MAX method, according to which the final form is obtained as a union of two fuzzy sets from Fig. 2 left. Fig. 2 on the right shows a graphic interpretation of accumulation according to the SUM method. Contours of the final shape are obtained as the algebraic sum of the contours in Fig. 3 right. If the sum is greater than one, then it is normalized to a value of one.
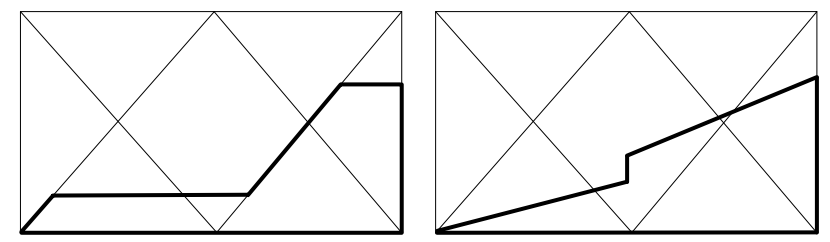

Figure 3 Accumulation phase (MAX - union, SUM - sum)
The resulting fuzzy set must be converted into a real number. That operation is called defuzzification. In Fig. 4 in the lower right corner, we see a bold full line representing number 30.8 on a scale of -100 to 100 . The resulting fuzzy set is defuzzified into real number 30.8 .

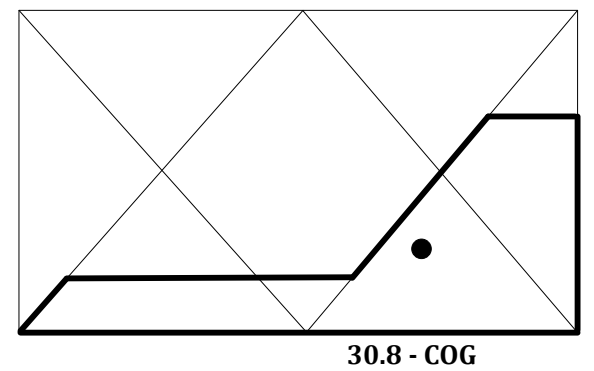

Figure 4 Defuzzification phase

Fuzzy systems can generally be divided into two large groups: Mamdani \& Sugenoor Takagi \& Sugeno, and so can the methods of defuzzification [3].

\section{FUZZY LOGIC SYSTEM FOR THE SELECTION OF BUILDING MATERIALS}

The concept described here creates the basis for modeling the given system of interdependence of the input criteria as a complex fuzzy system for the selection of building materials (Fig. 5). The basic concept of the model consists of four modules that are hierarchically organized on two levels, Fig. 5. The output size in the model represents the measure of priority of the materials, whereby each of the materials observed (straw, wood, brick and concrete/steel) is given a specific value of the criterion function, on the basis of which the user of the system selects the construction material.

The complex fuzzy system was designed so that the factors that are output on the first (lower) level (level I), are input on the upper level (level II). It can be seen in Fig. 5 that the first module of the first level (phase I of selecting the material) is influenced by the following factors: environmental impact, infrastructure equipment costs, site costs and risk of compromising the material. It is similar to the factors from module II and module III of the first level.

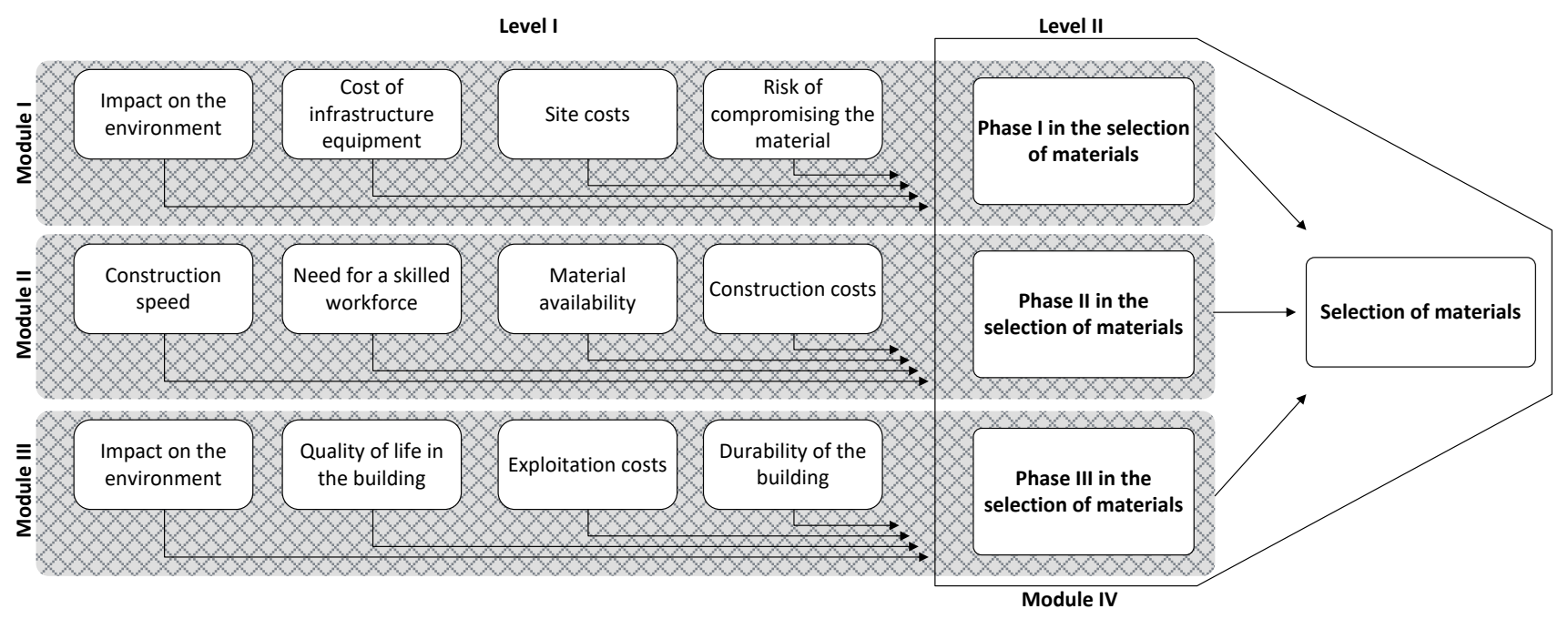

Figure $\mathbf{5}$ General model of the fuzzy system for selecting building materials 
The second module of the first level (phase II of the selection of materials) is influenced by the factors: construction speed, need for a skilled workforce, material availability and construction costs, while for the third module from the first level (phase III of the selection of materials) the influencing factors are: impact on the environment, quality of life in the building, exploitation costs, and durability of the building. The factors that influence the module at the second level of selection of material (level II) are: phases I, II and III of the material selection.

The fuzzy logic system for the selection of building materials was modeled through six phases: analyzing the problem, defining the linguistic values, selecting the membership function, forming a base of rules, selecting the methods of conclusion and defuzzification, and application of the fuzzy model. In the following section, the fuzzy model for selecting construction materials will be explained through the given phases.

\subsection{Analyzing the Problem}

Modeling a fuzzy logic system begins with a detailed analysis of the problem in order to determine the number of variables and their interdependence. Since this is a complex model that is implemented over three phases, and each phase has four criteria for modeling the system, it is divided into four subsystems (modules). The model is divided into modules because when designing complex systems, a person can manipulate well with a maximum of seven variables [24]. In this way the problem is reduced into four subsystems with four variables each.

The Fuzzy Logic System for the selection of building materials consists of three modules on the first level and one module on the second level. All modules from the first level have four input variables and one output variable (Fig. 6), while the module from the second level has three input and four output variables (Fig. 7).

The input variables of the modules from the first level are the criteria on the basis of which the materials are selected within phases I, II and III.

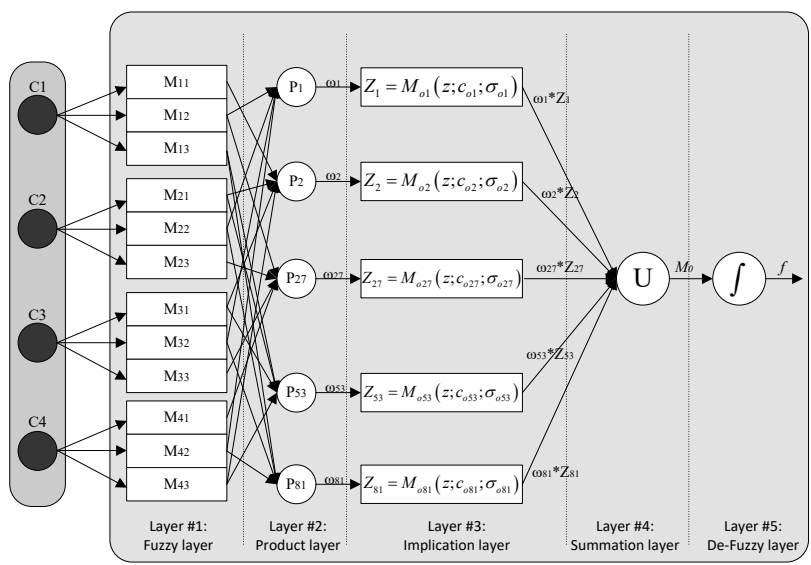

Figure 6 A general model of a fuzzy systemat level one (modules I, II and III)

Fig. 6 shows a fuzzy system from the first level with four fuzzy input variables and one output variable.

Each input variable is described with three linguistic variables, while the output variables are described with four linguistic variables. Fig. 7 shows the fuzzy system on the second level with three fuzzy input variables and four output variables. Each input variable is described with four linguistic variables, while the output variables are described with three linguistic variables.

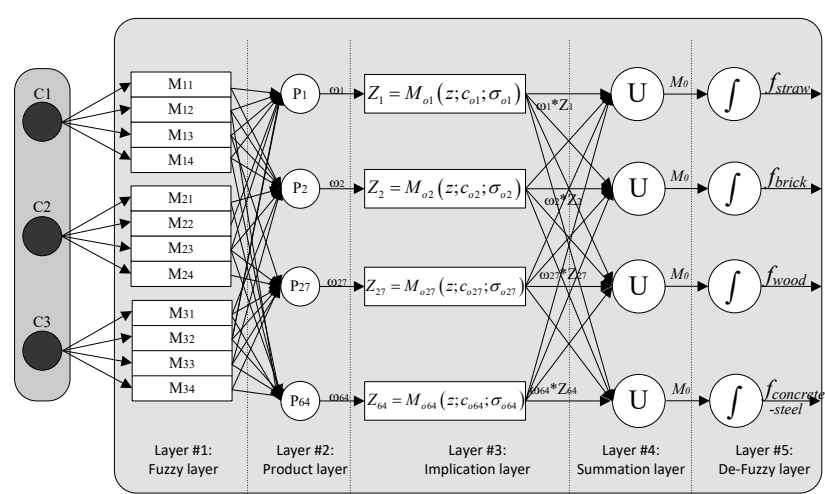

Figure $7 \mathrm{~A}$ general model of a fuzzy system at level two (module IV)

\subsection{Defining the Linguistic Values}

The linguistic variable, as it is seen from its name, takes its values from language. These values can be words or sentences of spoken language or artificially synthesized expressions. Linguistic variables are represented using fuzzy sets. All modules from the first level (module I, module II and module III) consist of four linguistic input variables:

- module I (phase I of material selection) linguistic input variables are impact on the environment, cost of infrastructure equipment, site costs and risk of compromising the material.

- module II (phase II of material selection) linguistic input variables are construction speed, need for a skilled workforce, material availability and construction costs.

- $\quad$ module III (phase III of material selection) linguistic input variables are impact on the environment, quality of life in the building, exploitation costs and durability of the building.

The module on the second level of material selection is made up of three linguistic input variables which are at the same time the output variables of each module from the first level. The four output variables from the second level of the module represent the construction materials from which the selection is made (straw, wood, brick and concrete/steel).

The values of the input variables are described by a set of linguistic descriptors $S=\left\{l_{1}, l_{2}, \ldots, l_{i}\right\}$, and $\epsilon H=\{0, \ldots$, $T$, where $T$ is the total number of linguistic descriptors. The linguistic variables are represented by a triangular fuzzy number defined as $(\alpha, \beta, \gamma)$, where $\beta$ is the value in which the membership function of the fuzzy number has its maximum value i.e. a value of 1.0. The values of $\alpha$ and $\beta$ represent the left and right distribution of the membership function of the value in which the membership function reaches its maximum value.

The number of linguistic descriptors, Fig. 8, is $T=10$ : unessential - U, unessential low - UL, very low - VL, fairly low - FL, low - L, medium - M, high - H, medium high $\mathrm{MH}$, very high - VH and perfect - $\mathrm{P}$.

After obtaining the linguistic values of the input variables, defuzzification of the fuzzy numbers is carried 
out for each criterion. Defuzzification of the linguistic descriptors is carried out using Eq. (3).

$$
\begin{aligned}
& g_{\alpha, \beta}(l)=\left[\beta \cdot f_{\alpha}\left(l_{k 1}\right)+(1-\beta) \cdot f_{\alpha}\left(l_{k 3}\right)\right] \\
& 0 \leq \beta \leq 1,0 \leq \alpha \leq 1
\end{aligned}
$$

where $f_{\alpha}\left(l_{k 3}\right)=l_{k 3}-\left(l_{k 3}-l_{k 2}\right) \cdot \alpha$ is the right limit of the confidence interval of fuzzy number $l$, while $f_{\alpha}\left(l_{k 1}\right)=\left(l_{k 2}-l_{k 1}\right) \cdot \alpha+l_{k 1}$ represents the left limit of the confidence interval of fuzzy number $l$. The value of $\alpha(0 \leq$ $\alpha \leq 1)$ represents the decision-maker's preference, while the value of $\beta(0 \leq \beta \leq 1)$ represents the pessimistic index of the decision-maker.

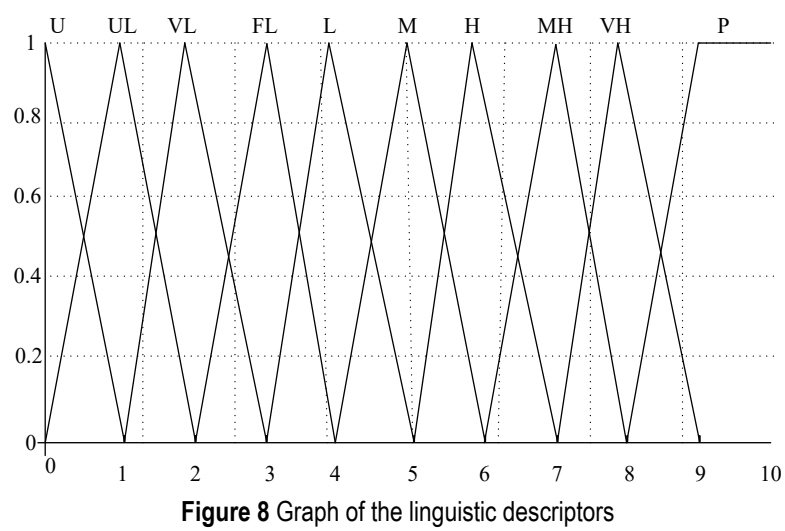

In this study, the extent of the confidence interval for each input variable is normalized as a numerical interval from 1 to 10 . After determining the confidence interval of the input variables it is necessary to determine the number and type of the membership functions. A large number of membership functions cause an increase in the number of rules, which can make the system more difficult to adjust. It is therefore recommended, in accordance with the nature of the variable, to begin with the smallest number of membership functions [28]. Reducing the number of membership functions must not affect the quality of the description of the variable. With this as the starting point, it was defined that in the model every input variable from the first level has three membership functions, while the output variables from the first level have four membership functions each.

There was no need for a large number of linguistic variables since this is an organizational system that does not require enormous precision in the way that fuzzy systems with automatic control do. A satisfactory level of precision of the system was reached with three or four linguistic values, as well as gradation when the output values were changed. On the other hand, it made the maximum number of rules to be 81 .

\subsection{Selecting the Membership Function}

In the initial phase of designing the system triangular functions were chosen as the membership functions. However, adjusting them did not enable enough precision and sensitivity of the system. Fig. 9 presents the sensitivity of the first module from the first level with triangular membership functions.

In Fig. 9 we see parts of the confidence interval of the output variables (flat sections of the diagram) where the fuzzy model was not sensitive and was inert. In addition, parts of the confidence interval were identified in which for small changes in the input values there are large changes in the output (steep sections of the diagram).
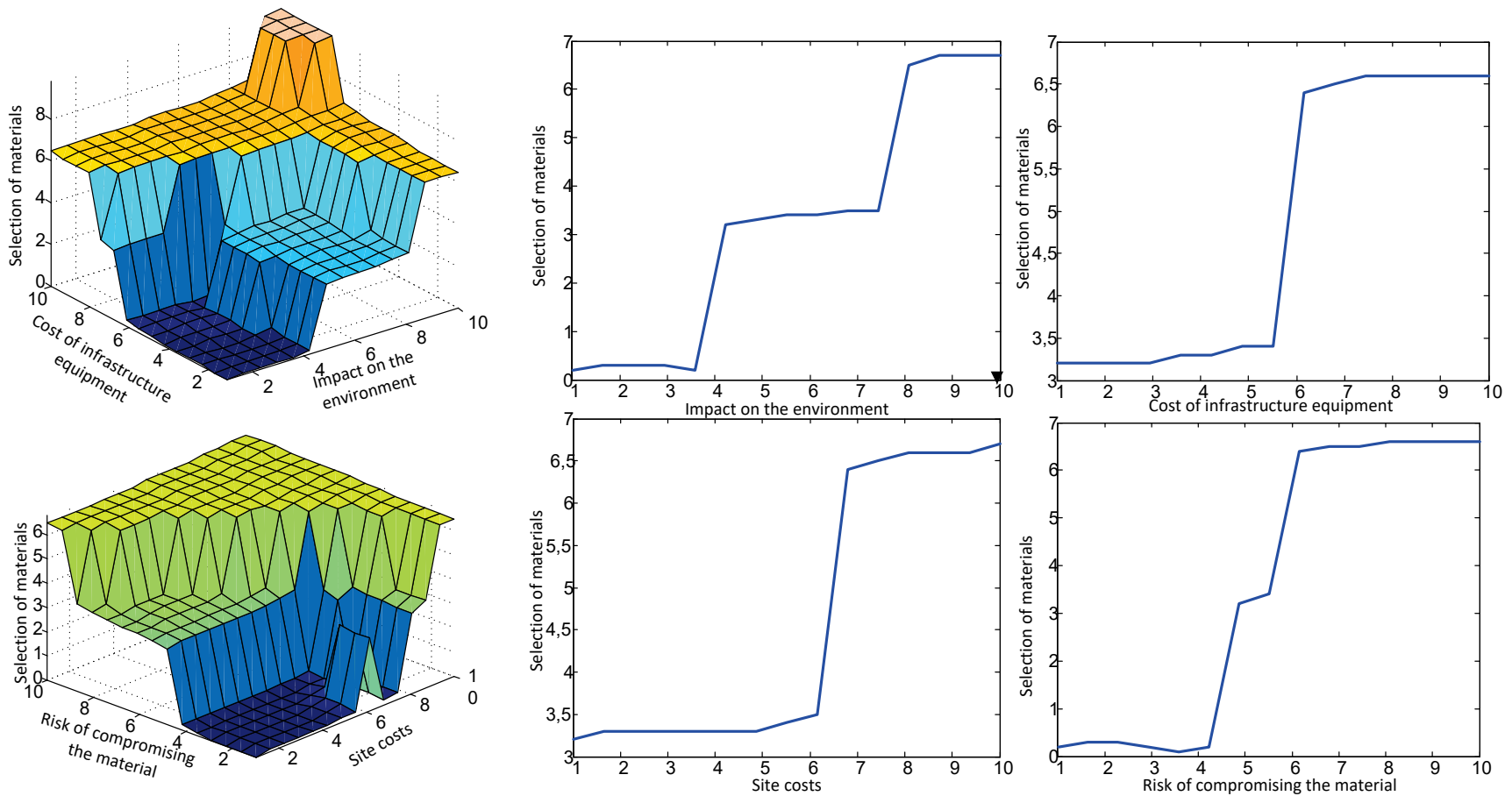

Figure 9 Sensitivity of the first module from the first level with triangular membership functions

In the next phase of adjusting the system within the fuzzy system, Gaussian curves were used. Gaussian functions provide a good description of the input and output variables of the modules from the first and second 
levels and secure a satisfactory sensitivity of the system. The sensitivity of the first module from the first level with Gaussian membership functions is shown in Fig. 10.
We can see in Fig. 10 that the system is sensitive in all parts of the confidence interval and that the Gaussian functions secure satisfactory gradation of the output.
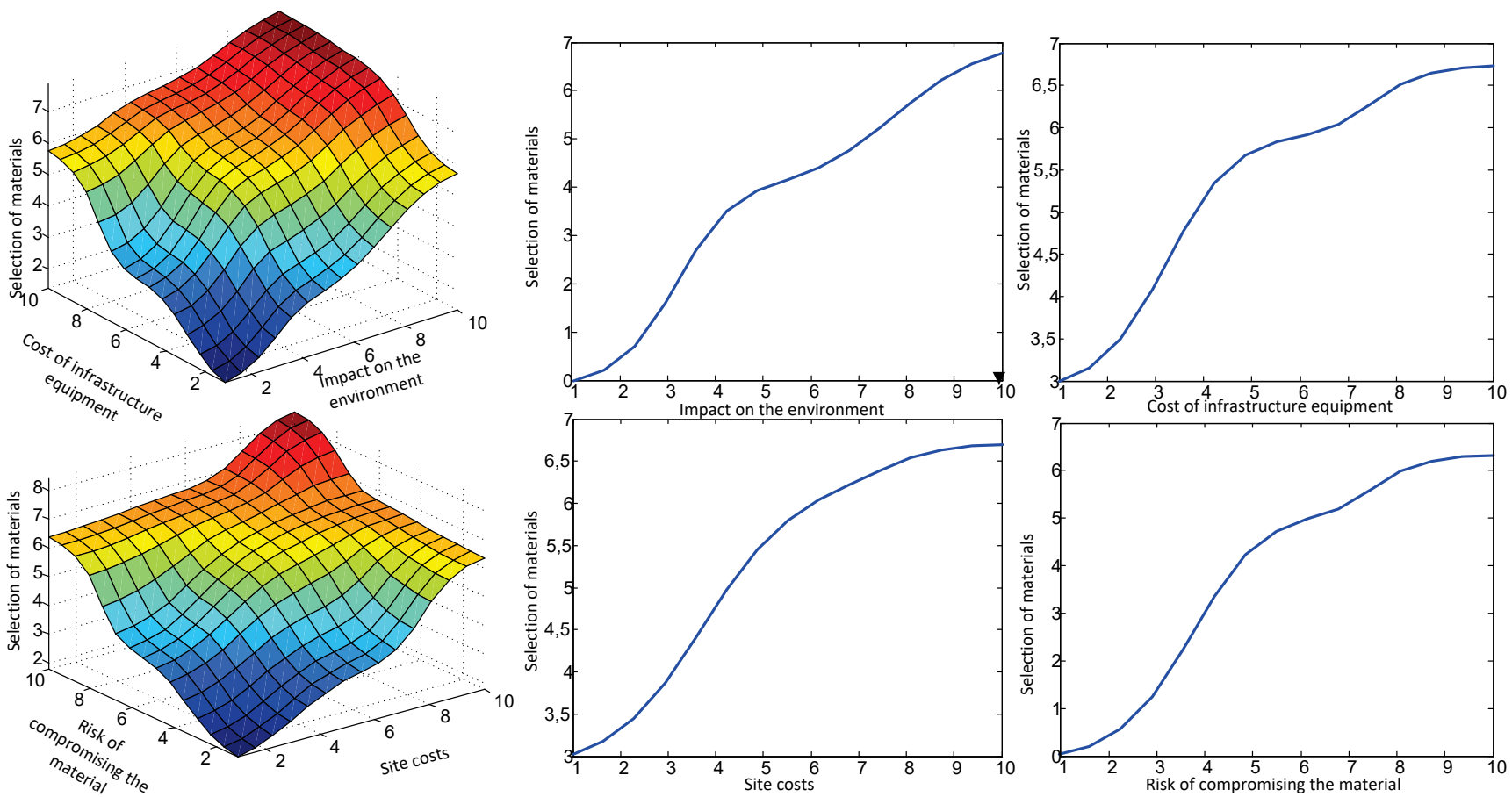

Figure 10 Sensitivity of the first module from the first level with Gaussian membership functions

Table 1 The membership function parameters of modules from the first level

\begin{tabular}{|c|c|c|c|c|}
\hline $\begin{array}{l}\text { Membership } \\
\text { function }\end{array}$ & FP 1 & FP 2 & FP 3 & FP 4 \\
\hline \multicolumn{5}{|c|}{ First module first level } \\
\hline $\begin{array}{l}\text { Impact on the } \\
\text { environment }\end{array}$ & $\begin{array}{c}{[1.368 ;} \\
1.357]\end{array}$ & $\begin{array}{c}{[1.872 ;} \\
5.64]\end{array}$ & $\begin{array}{l}{[1.715 ;} \\
8.983]\end{array}$ & - \\
\hline $\begin{array}{c}\text { Cost of } \\
\text { infrastructure } \\
\text { equipment } \\
\end{array}$ & {$[1.789 ; 1]$} & {$[1.75 ; 5.5]$} & $\begin{array}{l}{[1.062 ;} \\
8.979]\end{array}$ & - \\
\hline Site costs & $\begin{array}{l}{[2.151 ;} \\
1.715]\end{array}$ & $\begin{array}{c}{[1.773 ;} \\
5.595]\end{array}$ & $\begin{array}{l}{[1.287 ;} \\
9.547]\end{array}$ & - \\
\hline $\begin{array}{c}\text { Risk of } \\
\text { compromising the } \\
\text { material }\end{array}$ & $\begin{array}{l}\text { [1.503; } \\
2.143]\end{array}$ & $\begin{array}{c}{[1.648 ;} \\
5.5]\end{array}$ & $\begin{array}{l}0.999 ; \\
8.952]\end{array}$ & - \\
\hline Phase I & {$[1.416 ; 0]$} & $\begin{array}{l}\text { [1.416; } \\
3.333]\end{array}$ & $\begin{array}{c}{[1.416 ;} \\
6.667] \\
\end{array}$ & $\begin{array}{c}\text { [1.416; } \\
10]\end{array}$ \\
\hline
\end{tabular}

\begin{tabular}{|c|c|c|c|c|}
\multicolumn{7}{|c|}{ Second module first level } \\
\hline \multirow{2}{*}{ Construction speed } & {$[2.555 ;$} & {$[1.143 ;$} & {$[1.179 ;$} & - \\
& $1.455]$ & $5.836]$ & $8.362]$ & - \\
\hline \multirow{2}{*}{ Need for askilled } & {$[1.607 ;$} & {$[1.181 ;$} & {$[1.018 ;$} & - \\
workforce & $1.476]$ & $5.05]$ & $9.163]$ & - \\
\hline \multirow{2}{*}{ Material availability } & {$[1.365 ; 1]$} & {$[1.566 ; 6]$} & {$[1.341 ;$} & - \\
& & & $8.666]$ & - \\
\hline \multirow{2}{*}{ Construction costs } & {$[1.888 ;$} & {$[1.566 ;$} & {$[1.818 ;$} & - \\
& $2.503]$ & $5.881]$ & $8.269]$ & - \\
\hline \multirow{2}{*}{ Phase II } & {$[1.416 ; 0]$} & {$[1.416 ;$} & {$[1.416 ;$} & {$[1.416 ;$} \\
& & $3.333]$ & $6.667]$ & $10]$ \\
\hline
\end{tabular}

\begin{tabular}{|c|c|c|c|c|}
\hline \multicolumn{7}{|c|}{ Third module first level } \\
\hline Impact on the & {$[1.729 ;$} & {$[1.589 ;$} & {$[2.453 ;$} & - \\
environment & $1.595]$ & $5.05]$ & $9.352]$ & - \\
\hline Quality of life in the & {$[1.287 ;$} & {$[1.809 ;$} & {$[2.275 ;$} & - \\
building & $1.711]$ & $5.835]$ & $9.262]$ & - \\
\hline \multirow{2}{*}{ Exploitation costs } & {$[1.368 ;$} & {$[1.647 ;$} & {$[1.078 ;$} & - \\
& $1.857]$ & $4.47]$ & $9.019]$ & - \\
\hline Durability of the & {$[1.365 ;$} & {$[1.44 ;$} & {$[1.325 ;$} & - \\
building & $1.738]$ & $5.161]$ & $8.524]$ & - \\
\hline \multirow{2}{*}{ Phase II } & {$[1.416 ; 0]$} & {$[1.416 ;$} & {$[1.416 ;$} & {$[1.416 ;$} \\
& & $3.333]$ & $6.667]$ & $10]$ \\
\hline
\end{tabular}

Tab. 1 shows the parameters for the membership functions of the input and output variables of the modules from the first level. The first number represents the left and right distribution of the Gaussian curve along the abscissa, and the second number represents the value in which the Gaussian function has a value of 1 on the abscissa.

The membership function parameters for the output variables from the first level are shown in Tab. 2 .

Table 2 The membership function parameters for the second level

\begin{tabular}{|c|c|c|c|}
\hline Membership function (MF) & MF 1 & MF 2 & MF 3 \\
\hline Straw & 25 & 65 & 100 \\
\hline Wood & 35 & 65 & 100 \\
\hline Brick & 35 & 65 & 100 \\
\hline Concrete/steel & 25 & 65 & 100 \\
\hline
\end{tabular}

Since this is a fuzzy logic system, the Sugeno type of membership functions was described by a constant that is unique for one characteristic point of the confidence interval, while the remaining points of the interval have a value of zero.

\subsection{Forming the Base of Rules}

Linguistic rules are used as a link between the input and output of the fuzzy system modules. Expert knowledge on the process is expressed using a certain number of linguistic rules comprised of spoken words or artificial language. When it comes to complex systems, one of the big problems is that there is no standard or systematic method for transforming engineering knowledge or experience into fuzzy rules [24]. Neither is there a general procedure for choosing the optimum number of rules, since there are many factors affecting this decision, and it is very important for the speed of the system [29].

As we have already pointed out, for each module there are four linguistic input variables $(n=4)$, which are each 
described by three linguistic values - the membership functions $(M=3)$, and they can be combined in the base with a total of $M^{n}=3^{4}=81$ rules. This means that in our model, each module from the first level would have 81 rules (a total of 243 rules), while a module from the second level would have $M^{n}=3^{3}=27$ rules. Such a large number of rules would mean almost certain inconsistency in one of them, which would make it impossible to adjust the system.

There are numerous methods in the literature for developing an FLS base of rules from a known set of numerical data. These methods are processed in [30-32]. However, developing a base of FLS rules based on experience and intuition is a very complex process. In order to create a rule base, the analyst is forced to maintain lengthy communication with a large number of experts in the area of research. The characteristics of such a rule base depend on the number and quality of the experts, as well as the ability of the analysts to formulate their decisionmaking strategy. Due to the specificity of the model for selecting construction materials a new process was used for developing the FLS base: the method of aggregating the weights of rule premises (AWRP) [33].

\subsection{Selecting the Methods of Conclusion and Defuzzification}

The most commonly used methods of direct conclusion with the Mamdani type of FLS are the $M I N$ $M A X$ and PROD-SUM methods [34, 35]. In the initial phase of developing the system the $P R O D-S U M$ method of direct conclusion was used. This method is the usual choice when it is not important to manage the whole confidence interval of the output variables. However, in a large number of the model simulations in this case, the PRODSUM method proved to be unsuitable. One of the basic requirements was for the system to achieve a satisfactory level of sensitivity. This means that with certain small input changes, the output from the fuzzy system must also have these small changes in value, which could not be achieved using the PROD-SUM method.

By making adjustments it was not possible to achieve the desired form of the output criterion function of the FLS. Had this been achieved, it would have only been worthwhile for certain values of the input variables. By changing the parameters, the criterion function would look even less acceptable, and therefore the system would be even less sensitive. In some places where there should be a fall in the value of the function, there would be a rise. For this reason, the $M I N-M A X$ method was chosen to be the most suitable from those offered by the Matlab software package [36]. By selecting the $M I N-M A X$ method and adjusting the membership functions, the solutions obtained an acceptable form, which was also adopted.

The values of the input variables are fuzzified at the very beginning of the conclusion process in the fuzzy modules. Within the process of fuzzification, the membership functions defined for the input variables are applied to the actual values of the input variables, in order to determine the membership degree for the premise of each of the rules from the base [37]. The process of fuzzifying the input values of the fuzzy module will be explained using the example of the first module from the first level.
We will use the example that the input variable from the first module of the first level impact on the environment is described with the linguistic descriptor VH (Very high), cost of infrastructure equipment is described with the linguistic descriptor P (Perfect), site costs are described with the linguistic descriptor MH (Medium High) and risk of compromising the material is described with the linguistic descriptor $M$ (Medium). After obtaining these values, the expert system carries out fuzzification of the input variables. Each variable is made up of three fuzzy sets and the purpose of fuzzification is to determine to which fuzzy set the input variable belongs, and to express this belonging with a numerical value in the domain $[0 ; 1]$. By fuzzifying the value of impact on the environment the values are obtained for belonging to the variable impact on the environment shown in Fig. 11.

Fuzzification of the input variables for the second and third modules from the first level and the fuzzy modules from the second level is carried out in the same way, and so we will not enter into a more detailed explanation of the fuzzification of the input variables for the remaining fuzzy modules.
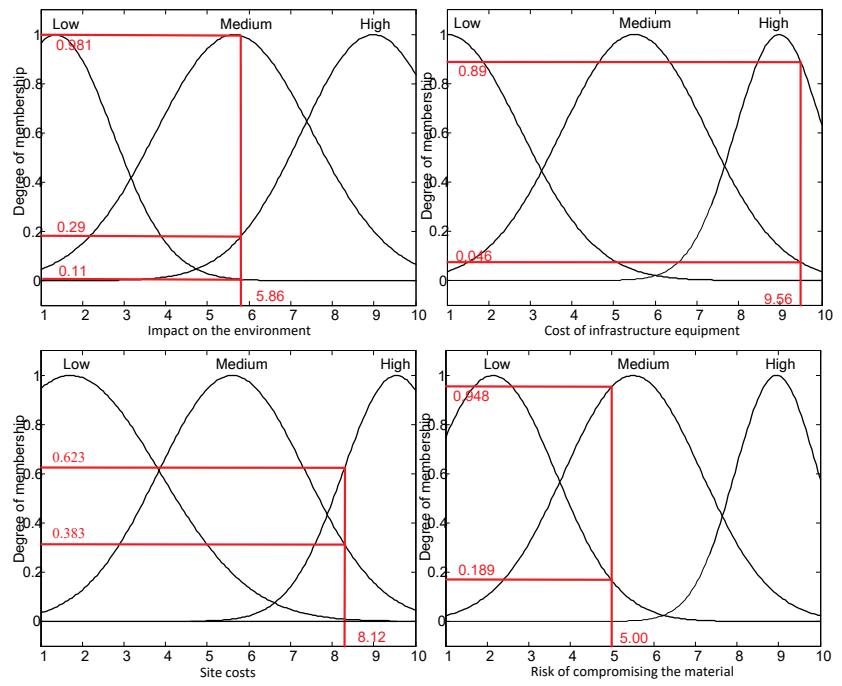

Figure 11 Fuzzification of the input variables of the first module from the first level

After fuzzification of the input values there follows an analysis of the values and their comparison with the sets of rule premises from the base of rules. In this example, analysis confirmed that rules 2, 8, 10 and 13 were activated. Each rule gives its own intermediate result, which can be represented by a corresponding fuzzy set.

Rule number 2 is the first to be carried out: "IF impact on the environment is Medium ${ }^{\wedge} \operatorname{cost}$ of infrastructure equipment is High^site costs are Low^risk of compromising the material is Low THEN the selection of material is Wood". In Fig. 11 it can be seen that by defuzzifying linguistic descriptor $\mathrm{H}$ (High) a real number of 5.86 is obtained, which in the framework of the fuzzy set Medium impact on the environment corresponds to the value 0.981 . Also, from Fig. 11 it can be seen that:

- If cost of infrastructure equipment is represented by linguistic descriptor P (Perfect), by defuzzifying the linguistic descriptor a value of 9.56 is obtained, which in the fuzzy set High expertise corresponds to the value 0.89 . 
- If site costs are represented by linguistic descriptor MH (Medium High), by defuzzifying the linguistic descriptor a value of 8.12 is obtained, which in the fuzzy set Medium site costs corresponds to the value 0.383 .

- If risk of compromising the material is represented by linguistic descriptor M (Medium), by defuzzifying the linguistic descriptor a value of 5.00 is obtained, which in the fuzzy set Low risk of compromising the material corresponds to the value 0.189 .

Since the operator "and" $(\wedge)$ is used between the antecedents of the rules, in order for all four conditions to be met the lower value is taken, that is, the intersection of the fuzzy sets, which in this case is 0.189 . The value obtained in this way is transferred to the fuzzy set that represents the conclusion. In this specific case the fuzzy set Wood is a possible answer. This result is shown graphically in Fig. 12a.

Secondly, rule number 8 is carried out: "IF impact on the environment is Medium $\wedge$ cost of infrastructure equipment is High $\wedge$ site costs are Low $\wedge$ risk of compromising the material is Medium THEN the selection of material is brick". The intermediate result after carrying out this rule is given by the fuzzy set in Fig. 12b.
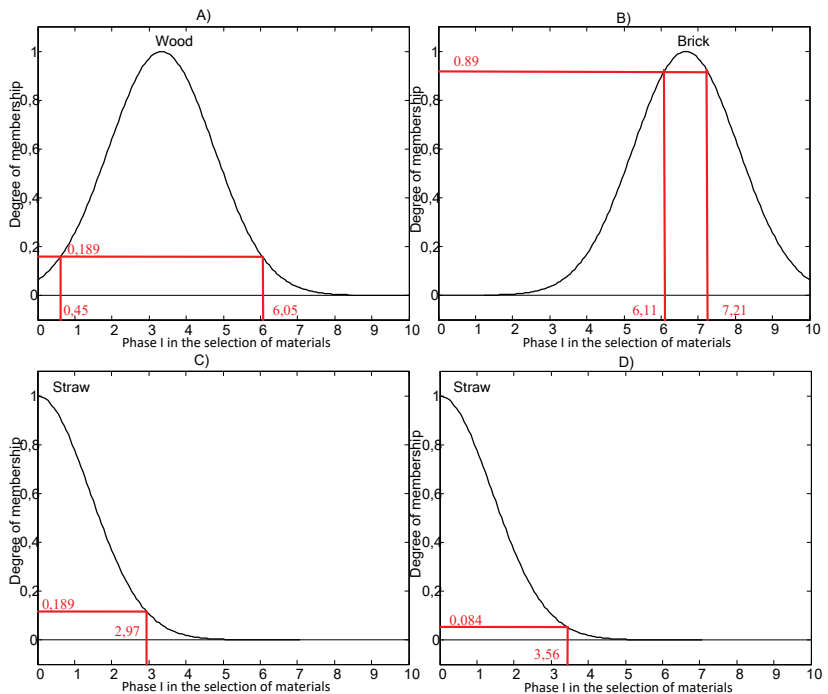

Figure 12 Graphic presentations of the intermediate results after carrying out rules $2,8,10$ and 13
Thirdly, rule number 10 is carried out: "IF impact on the environment is Medium ${ }^{\wedge}$ cost of infrastructure equipment is High $\wedge$ site costs are Low $\wedge$ risk of compromising the material is Low THEN the selection of material is straw". The intermediate result after carrying out this rule is given by the fuzzy set in Fig. 12c.

Fourthly, rule number 13 is carried out. This rule states "IF impact on the environment is Low $\wedge$ cost of infrastructure equipment is Medium ${ }^{\wedge}$ site costs are Low ${ }^{\wedge}$ risk of compromising the material is Low THEN the selection of material straw". The intermediate result after carrying out this rule is given by the fuzzy set in Fig. 12d.

The union operation is performed on the fuzzy sets and the resulting fuzzy set is at the same time the result of the conclusion of the first module from the first level. When we apply the union operation to the fuzzy sets from Fig. 12 we obtain the resulting fuzzy set shown in Fig. 13.

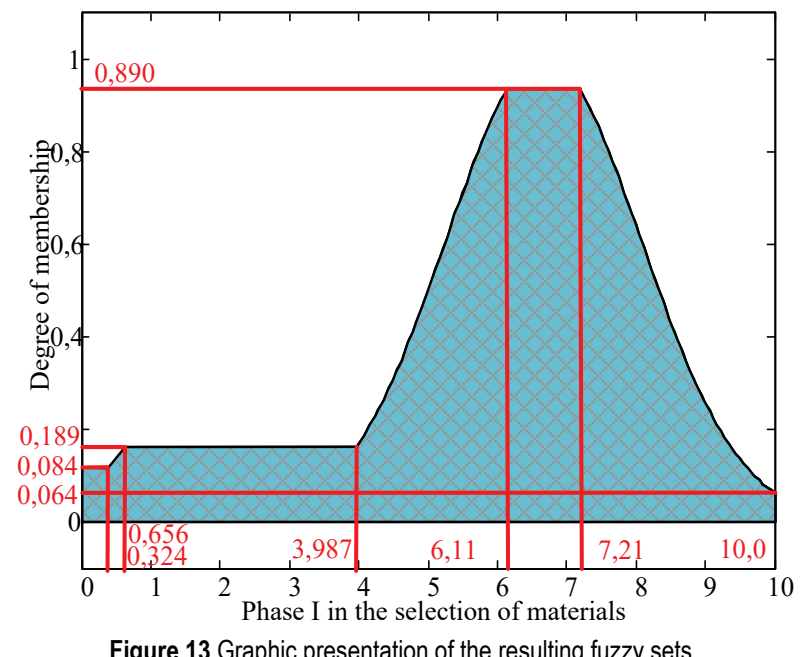

The center of gravity method was chosen as the method of defuzzification, since it is usual and suitable for developing this kind of fuzzy system, as it ensures the necessary continuity and gradation of the output. By using this method of defuzzification, we obtain the final value of the criterion function of the first module from the first level:

$$
u=\frac{\sum_{i=1}^{n} \mu\left(x_{i}\right) \cdot x_{i}}{\sum_{i=1}^{n} \mu\left(x_{i}\right)}=\frac{0.084 \cdot 0.324+0.189 \cdot 0.656+0.189 \cdot 3.987+0.890 \cdot 6.11+0.8907 .21+0.064 \cdot 10}{0.084+0.189+0.189+0.890+0.890+0.064}=5.811
$$

The resulting fuzzy sets for the remaining two modules from the first level are obtained in the same way. In contrast to the modules from the first level which contain Mamdani type FLS, the fuzzy module from the second level was developed as a Sugenotype FLS. Since this is a specific FLS and it is the resulting module in which the final selection of building materials is made, the next section will show the process of fuzzification of the input values of the module from the second level and the selection of building materials.

The values obtained by the defuzzification of the resulting fuzzy sets of the modules from the first level represent the input data that need to be fuzzified in the module from the second level. Fuzzification of the input data from the second level module is shown in Fig. 14. Fuzzification of the input values, as in the previous case, is followed by an analysis of the values and their comparison with the set of rules premises from the base of rules. Analysis determined that rules 1, 12 and 17 were active. Each rule gives its own intermediate result which can be represented by the appropriate fuzzy set. Fig. 15 presents the resulting fuzzy sets according to the materials. 

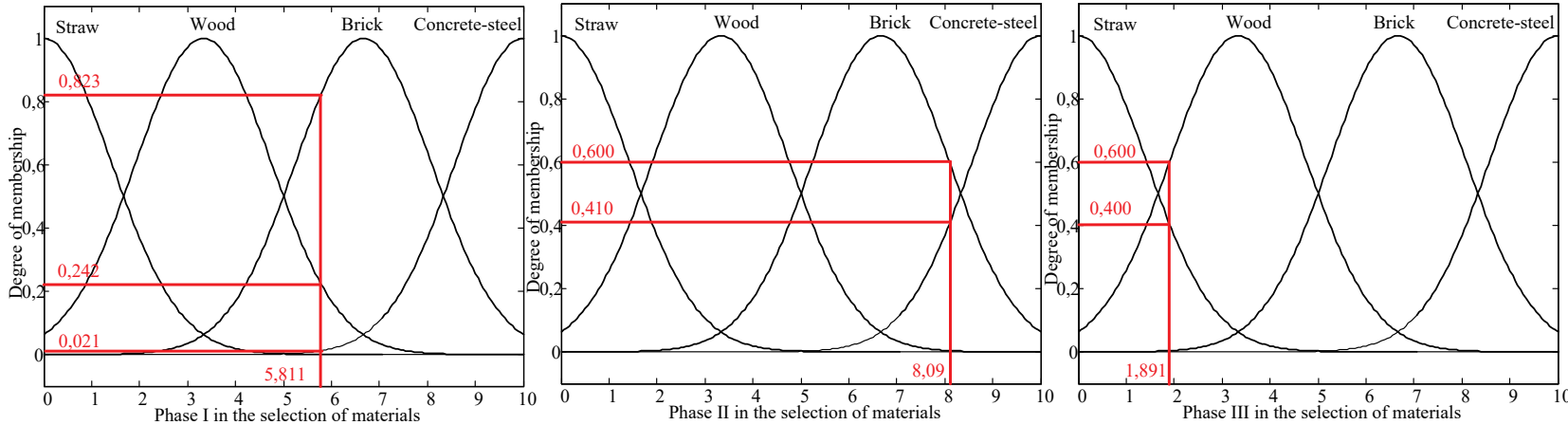

Figure 14 Fuzzification of the input variablesof the module from the second level-the resulting FLS
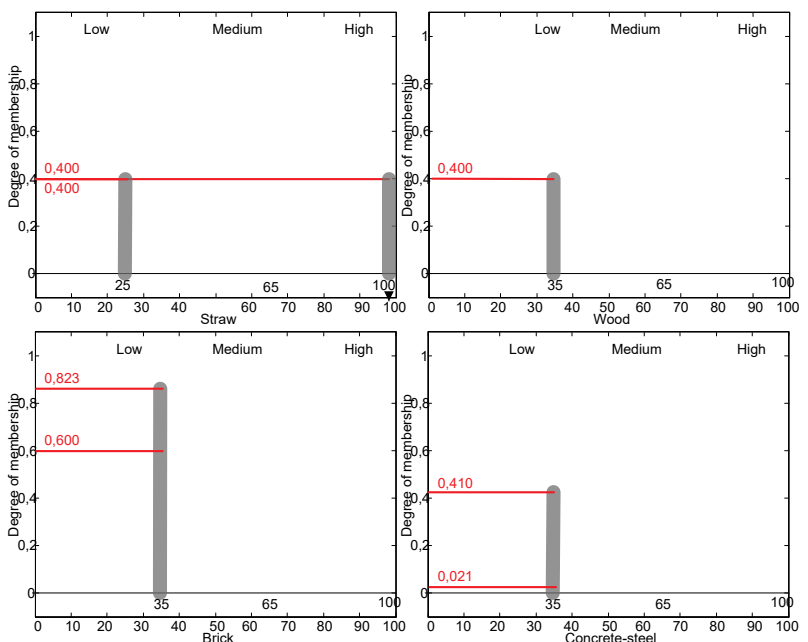

Figure 15 Graphs of the resulting fuzzy sets by material

As with the modules from the first level, the method chosen for defuzzification was the center of gravity method, since it ensures the necessary continuity and gradation of output. By applying this defuzzification method we obtain the final values of the preference for construction materials. In this example brick is chosen as the building material because it has the highest preference index which is:

Opeka $=\frac{\sum_{i=1}^{n} \mu\left(x_{i}\right) \cdot x_{i}}{\sum_{i=1}^{n} \mu\left(x_{i}\right)}=\frac{0.823 \cdot 35+0.600 \cdot 35}{0.35+0.35}=71.15 \%$

The connection between the fuzzy modules from the first level and the module from the second level is established by the program code:

$\mathrm{a}=$ evalin('base','input1');

$\mathrm{b}=$ evalin('base','input2');

$\mathrm{c}=$ evalin('base','input3');

$\mathrm{d}=$ evalin('base','input4');

$\mathrm{e}=$ readfis('Ifaza.fis');

$\mathrm{fl}=$ evalfis $([\mathrm{a} b \mathrm{c} d], \mathrm{e})$;

assignin('base','fl',fl),

if $\mathrm{f} 1<=1,3$

set(handles.edit1,'String','Slama');

assignin('base','input12','Slama');

elseif and(fl $>1,3, \mathrm{fl}<=2)$

set(handles.edit1,'String','Slama iliDrvo');

assignin('base', 'input12','Slama iliDrvo');

elseif and(f1>2, $\mathrm{fl}<=4,4)$

set(handles.edit1,'String','Drvo'); assignin('base', 'input12','Drvo');

elseif and(f1>4,4, $\mathrm{fl}<=5,5)$

set(handles.edit1,'String','Drvo iliOpeka');

assignin('base', 'input12','Drvo iliOpeka')

elseif and(f1>5,5, $\mathrm{fl}<=7,5)$

set(handles.edit1,'String','Opeka');

assignin('base', 'input12','Opeka');

elseif and(fl>7,5, fl<=8,5)

set(handles.edit1,'String','Opeka iliBeton/Celik');

assignin('base', 'input12','Opeka iliBeton/Celik');

elseif $\mathrm{fl}>8,5$

set(handles.edit1,'String','Beton/Celik'); assignin('base', 'input12','Beton/Celik');

a=evalin('base','Ifaza');

b=evalin('base', 'IIfaza');

$\mathrm{c}=$ evalin('base', 'IIIfaza');

$\mathrm{e}=$ readfis('Main.fis');

$\mathrm{k}=$ evalfis $([\mathrm{a} \mathrm{b} \mathrm{c}], \mathrm{e})$;

assignin('base','k',k), set(handles.edit4,'String',k(1:1)); set(handles.edit5,'String', $\mathrm{k}(2: 2))$; set(handles.edit7,'String',k(3:3)); set(handles.edit8,'String',k(4:4));

end

\section{APPLICATION OF THE MODEL}

This step is a logical phase in the life cycle of the model. The model should be applied and corrections, adjustments and improvements made as necessary. For this model, the user form was developed in the Matlab software package. Entering "IM" in the command line of the Matlab software package launches the user program for the selection of building materials (Fig. 16).

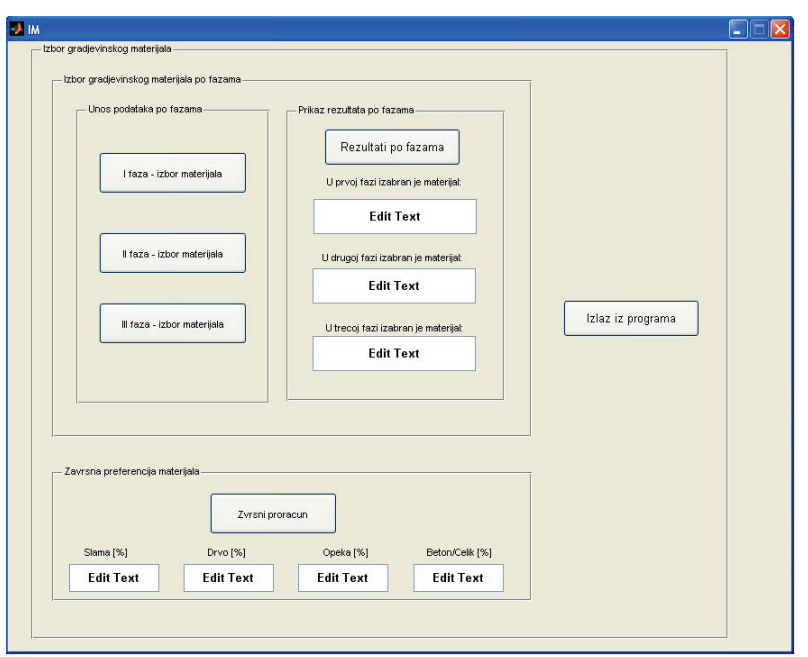

Figure 16 User program for the selection of building materials 
By activating the programming key phase $I$ - the selection of materials the fuzzy module from the first level is launched and the values of the criteria from the first module are entered. The values of the criteria are normalized to the interval 1-10. The user chooses a numerical value for every criterion, which the software assigns to the corresponding input criterion of the FLS (Fig. 17).

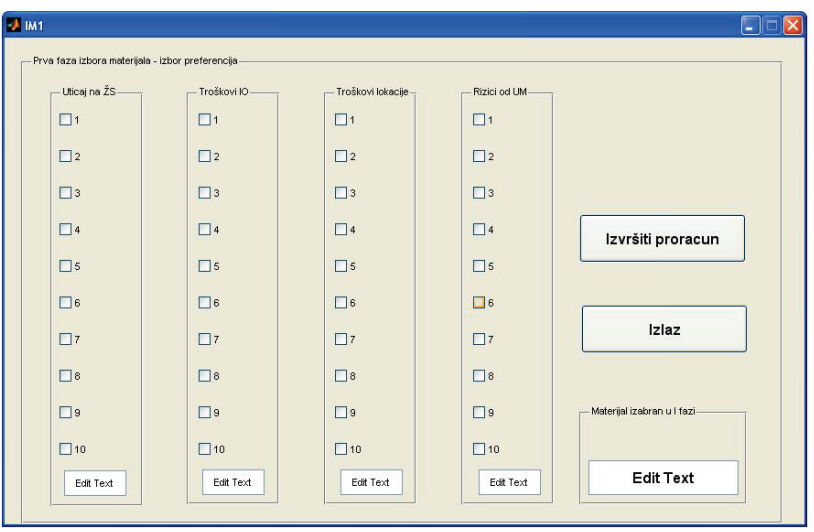

Figure 17 Data entry to the first module of the first level

By pressing the program button Calculate we obtain the recommendation for the building material selected in phase I of selection of materials.

After defining the construction material in phase I, by pressing the program button phase II - selection of materials and phase III - selection of materials the second and third fuzzy modules from the first level are launched and the values of the criteria in the modules are entered. The values of the criteria, as in the first module, are normalized to an interval of 1-10. The user chooses a numerical value for every criterion, which the software assigns to the corresponding input criterion of the FLS. By pressing the program button Calculate we obtain a recommendation for the construction materials for phases II and III in the selection of materials.

After determining the construction materials by phases, pressing the program button Final calculation begins the fuzzy module from the second level with the data obtained in phases I, II and III in the selection of materials. After completing the fuzzy module from the second level the final preferences for the materials are obtained, Fig. 18.

The model was tested on the case of selecting construction materials for constructing residential buildings at five locations. The results are shown in Tab.3.

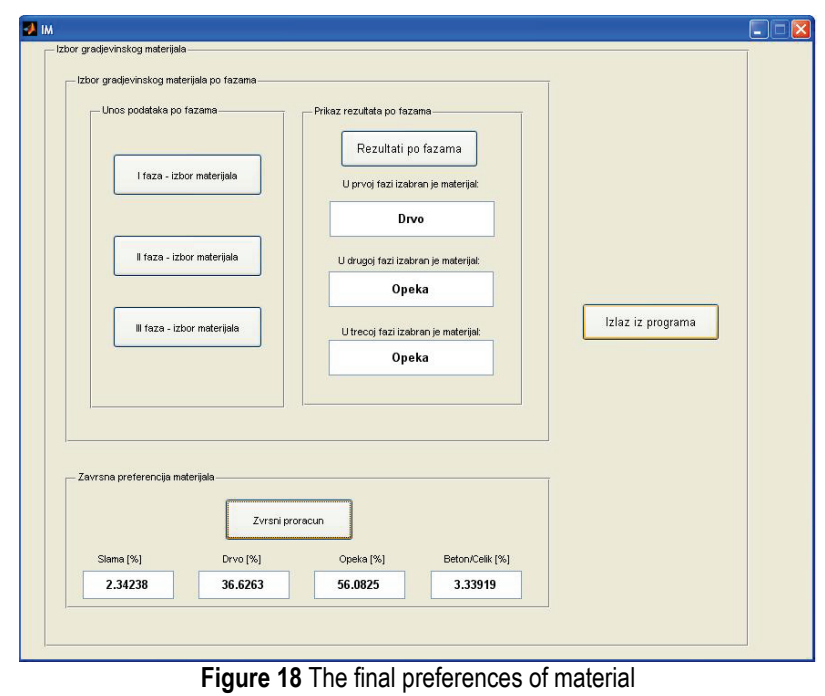

Table 3 Selection of construction material using a modular fuzzy logic system

\begin{tabular}{|c|c|c|c|c|c|c|c|}
\hline \multirow{2}{*}{ Location } & \multicolumn{3}{|c|}{ Input values } & \multicolumn{4}{|c|}{ Recommendation for material } \\
\cline { 2 - 8 } & Phase I & Phase II & Phase III & Straw & Wood & Brick & Concrete/ steel \\
\hline L1 & {$[4 ; 7 ; 3 ; 5]$} & {$[1 ; 4 ; 2 ; 1]$} & {$[8 ; 3 ; 1 ; 1]$} & 0.25 & 40.26 & 57.70 & 1.30 \\
\hline L2 & {$[1 ; 2 ; 2 ; 2]$} & {$[9 ; 4 ; 4 ; 9]$} & {$[6 ; 3 ; 5 ; 1]$} & 17.9 & 27.30 & 48.50 & 0.40 \\
\hline L3 & {$[8 ; 10 ; 10 ; 9]$} & {$[8 ; 6 ; 8 ; 9]$} & {$[5 ; 6 ; 8 ; 10]$} & 0.12 & 23.00 & 46.50 & 26.20 \\
\hline L4 & {$[7 ; 8 ; 1 ; 3]$} & {$[8 ; 5 ; 6 ; 9]$} & {$[2 ; 2 ; 4 ; 2]$} & 0.90 & 56.90 & 41.40 & 0.320 \\
\hline L5 & {$[1 ; 2 ; 2 ; 1]$} & {$[2 ; 2 ; 3 ; 1]$} & {$[1 ; 10 ; 2 ; 1]$} & 43.60 & 27.90 & 25.70 & 0.20 \\
\hline
\end{tabular}

The results shown in Tab. 3 represent the preferences for construction materials for selected locations. The preferences for construction materials in the modular fuzzy logic system are in the interval $[0 ; 100]$. The construction material that has the greatest preference has an advantage over construction materials with the least preference. For example, at location 5 (L5) the greatest preference is for straw (43.60), while the least preference is for concrete/steel $(0.20)$.

\section{CONCLUSION}

The paper offers a new evaluation and prediction model for the selection of building materials, using a fuzzy method based on fuzzy logic. The authors believe that the new approach to the selection of building materials allows for a considerable qualitative shift in the methodology applied to facilitate the choice.

This model expands the theoretical framework of knowledge in the area of choosing construction materials, since it is a new model that considers the selection of construction materials in an original way. The existing problem is considered using new methodology, which creates the basis for a further theoretical, but also practical upgrade.

Also, this model highlights criteria that have not been considered in the models so far, and are of importance for this problem. By introducing new criteria and presenting them in the model, the need for their consideration in further analysis of this and other similar problems is highlighted.

The fuzzy model presented in this paper has three main advantages over other methods. First, it may reflect a variety of decision-making criteria if required. The quality of adaptability is inherent in the system, as it is possible to adjust a fuzzy rule base. Fuzzy inference rules are very important for building materials selection, more notably so within the descriptive approach, as it prefers an intuitive, heuristic search for solutions in the selection process. The flexibility of the model overcomes the restrictions of conventional evaluation models, prioritizing building materials by simply aggregating the results of the 
evaluation criteria. Second, the model presented in this paper is efficient under conditions of uncertainty, supporting decision makers in the selection of building materials. Third, it can be implemented as a computerbased system, thus supporting a dynamic decision-making process in a building materials selection. The model allows for a fairly quick and objective assessment of cost and risk factors in the selection of building materials in a changeable environment.

A natural direction for future research would be to identify additional parameters affecting the selection of building materials, and implement additional decisionmaking criteria in the model. Along these lines, fuzzy linear and dynamic programming methods, combined with heuristic and metaheuristic methods, are carving themselves a proper application niche.

\section{REFERENCES}

[1] Pamučar, D. \& Ćirović, G. (2015). The selection of transport and handling resources in logistics centres using MultiAttributive Border Approximation area Comparison (MABAC). Expert Systems with Applications, 42, 30163028. https://doi.org/10.1016/j.eswa.2014.11.057

[2] Lombera, J. T. S. J. \& Rojo, J. C. (2010). Industrial building design stage based on a system approach to their environmental sustainability. Construction and Building Materials, 24(4), 438-447. https://doi.org/10.1016/j.conbuildmat.2009.10.019

[3] Pujadas, P., Pardo-Bosch, F., Aguado-Renter, A., \& Aguado, A. (2017). MIVES multi-criteria approach for the evaluation, prioritization, and selection of public investment projects. $A$ case study in the city of Barcelona. Land Use Policy, 64, 2937. https://doi.org/10.1016/j.landusepol.2017.02.014

[4] del Caño, A., Gómez, D., \& de la Cruz, M. P. (2012). Uncertainty analysis in the sustainable design of concrete structures: A probabilistic method. Construction and Building Materials, 37, 865-873.

https://doi.org/10.1016/j.conbuildmat.2012.04.020

[5] De la Fuente, A., Pons, O., Josa, A., \& Aguado, A. (2016). Multi-Criteria Decision Making in the sustainability assessment of sewerage pipe systems. Journal of cleaner production, 112, 4762-4770. https://doi.org/10.1016/j.jclepro.2015.07.002

[6] Akhtar, S., Reza, B., Hewage, K., Shahriar, A., Zargar, A., \& Sadiq, R. (2015). Life cycle sustainability assessment (LCSA) for selection of sewer pipe materials. Clean Technologies and Environmental Policy, 17(4), 973-992. https://doi.org/10.1007/s10098-014-0849-x

[7] De la Fuente, A., Blanco, A., Armengou, J., \& Aguado, A. (2017). Sustainability based-approach to determine the concrete type and reinforcement configuration of TBM tunnels linings. Case study: Extension line to Barcelona Airport T1. Tunnelling and Underground Space Technology, 61, 179-188. https://doi.org/10.1016/j.tust.2016.10.008

[8] Pons, O. \& de la Fuente, A. (2013). Integrated sustainability assessment method applied to structural concrete columns. Construction and Building Materials, 49, 882-893. https://doi.org/10.1016/j.conbuildmat.2013.09.009

[9] Pujadas, P., Pardo-Bosch, F., Aguado-Renter, A., \& Aguado, A. (2017). MIVES multi-criteria approach for the evaluation, prioritization, and selection of public investment projects. $A$ case study in the city of Barcelona. Land Use Policy, 64, 2937. https://doi.org/10.1016/j.landusepol.2017.02.014

[10] Rashidi, M., Ghodrat, M., Samali, B., Kendall, B., \& Zhang, C. (2017). Remedial modelling of steel bridges through application of analytical hierarchy process (AHP). Applied Sciences, 7(2), 168. https://doi.org/10.3390/app7020168
[11] Jia, J., Ibrahim, M., Hadi, M., Orabi, W., \& Xiao, Y. (2018). Multi-Criteria Evaluation Framework in Selection of Accelerated Bridge Construction (ABC) Method. Sustainability, 10(11), 4059. https://doi.org/10.3390/su10114059

[12] Formisano, A. \& Mazzolani, F. M. (2015). On the selection by MCDM methods of the optimal system for seismic retrofitting and vertical addition of existing buildings. Computers \& Structures, 159, 1-13. https://doi.org/10.1016/j.compstruc.2015.06.016

[13] Terracciano, G., Di Lorenzo, G., Formisano, A., \& Landolfo, R. (2015). Cold-formed thin-walled steel structures as vertical addition and energetic retrofitting systems of existing masonry buildings. European Journal of Environmental and Civil Engineering, 19(7), 850-866. https://doi.org/10.1080/19648189.2014.974832

[14] Šiožinyte, E., Antuchevičienè, J., \& Kutut, V. (2014). Upgrading the old vernacular building to contemporary norms: multiple criteria approach. Journal of Civil Engineering and Management, 20(2), 291-298. https://doi.org/10.3846/13923730.2014.904814

[15] Ozcan-Deniz, G. \& Zhu, Y. (2015). A multi-objective decision-support model for selecting environmentally conscious highway construction methods. Journal of Civil Engineering and Management, 21(6), 733-747. https://doi.org/10.3846/13923730.2014.893915

[16] Stević, Ž., Pamučar, D., Subotić, M., Antuchevičiene, J., \& Zavadskas, E. (2018). The location selection for roundabout construction using rough BWM-rough WASPAS approach based on a new rough hamy aggregator. Sustainability, 10(8), 2817. https://doi.org/10.3390/su10082817

[17] Rashid, K., Razzaq, A., Ahmad, M., Rashid, T., \& Tariq, S. (2017). Experimental and analytical selection of sustainable recycled concrete with ceramic waste aggregate. Construction and Building Materials, 154, 829-840. https://doi.org/10.1016/j.conbuildmat.2017.07.219

[18] Raslanas, S., Kliukas, R., \& Stasiukynas, A. (2016). Sustainability assessment for recreational buildings. Civil Engineering and Environmental Systems, 33(4), 286-312. https://doi.org/10.1080/10286608.2016.1236086

[19] Tsai, W. H., Lin, S. J., Lee, Y. F., Chang, Y. C., \& Hsu, J. L. (2013). Construction method selection for green building projects to improve environmental sustainability by using an MCDM approach. Journal of Environmental Planning and Management, 56(10), 1487-1510. https://doi.org/10.1080/09640568.2012.731385

[20] Zavadskas, E. K., Vainiūnas, P., Turskis, Z., \& Tamošaitienè, J. (2012). Multiple criteria decision support system for assessment of projects managers in construction. International journal of information technology \& decision making, 11(02), 501-520. https://doi.org/10.1142/S0219622012400135

[21] Mardani, A., Zavadskas, E. K., Khalifah, Z., Jusoh, A., \& Nor, K. M. (2016). Multiple criteria decision-making techniques in transportation systems: a systematic review of the state of the art literature. Transport, 31(3), 359-385. https://doi.org/10.3846/16484142.2015.1121517

[22] Herrera, F. (2008). Genetic fuzzy systems: taxonomy, current research trends and prospects. International Journal of Uncertainty, Fuzziness and Knowledge-Based Systems, 27-46. https://doi.org/10.1007/s12065-007-0001-5

[23] Ćirović, G. \& Pamučar, D. (2013). Decision support model for prioritizing railway level crossings for safety improvements: Application of the adaptive neuro-fuzzy system. Expert Systems with Applications, 40(6), 2208-2223. https://doi.org/10.1016/j.eswa.2012.10.041

[24] Jovanović A., Pamučar D., \& Pejčić-Tarle S. (2014). Green vehicle routing in urban zones - A neuro-fuzzy approach. Expert systems with applications, 41, 3189-3203. https://doi.org/10.1016/j.eswa.2013.11.015 
[25] Ngamchai, S. \& Lovell, D. J. (2003). Optimal time transfer in bus transit route network design using a genetic algorithm. Journal of Transportation Engineering, 129(5), 510-521. https://doi.org/10.1061/(ASCE)0733-947X(2003)129:5(510)

[26] Kuo, Y. \& Wang, C. C. (2011). Optimizing the vrp by minimizing fuel consumption. Management of Environmental Quality: An International Journal, 22(4), 440- 450. https://doi.org/10.1108/14777831111136054

[27] Fan, W. \& Machemehl, R. B. (2006). Optimal transit route network design problem with variable transit demand: genetic algorithm approach. Journal of Transportation Engineering, 132(1), 40-51. https://doi.org/10.1061/(ASCE)0733-947X(2006)132:1(40)

[28] Kandel, A., Chew, G., \& Schneider, M. (1994). Designing Fuzzy Inference Procedures. The second World Congress on Expert Systems, Lisbon.

[29] Mamdani, E. H. \& Assilian, S. (1974). Application of Fuzzy Algorithms for Control of Simple Dynamic Plant. Proceedings of the Institute of Electrical Engineers 121, 1585-1588. https://doi.org/10.1049/piee.1974.0328

[30] Miller, G. A. (1956). The magical number seven plus or minus two: some limits on our capacity for processing information. Psychological Review, 63, 81-97. https://doi.org/10.1037/h0043158

[31] Ravi, V., Reddy, P. J., \& Zimmermann H. J. (2001). Fuzzy rule base generation for classification and its minimization via modified threshold accepting. Fuzzy Sets and System, 120(2), 271-279. https://doi.org/10.1016/S0165-0114(99)00100-1

[32] Wang L. X. \& Mendel J. M. (1992). Generating fuzzy rules by learning from examples. IEEE Transactions on Systems, Man, and Cybernetics, 22(6), 1414-1427. https://doi.org/10.1109/21.199466

[33] Božanić, D. \& Pamučar, D. (2014). Developing a rule base for a fuzzy logic decision support system by aggregating the weights of rules premises AWR. Tehnika, 1, 129-138. https://doi.org/10.5937/tehnika1401129B

[34] Sremac, S., Tanackov, I., Kopic, M., \& Radovic, D. (2018). ANFIS model for determining the economic order quantity. Decision Making: Applications in Management and Engineering, 1(2), 81-92. https://doi.org/10.31181/dmame1802079s

[35] Stojčić, M., Stjepanović, A., \& Stjepanović, Đ. (2019). ANFIS model for the prediction of generated electricity of photovoltaic modules. Decision Making: Applications in Management and Engineering, 2(1), 35-48. https://doi.org/10.31181/dmame1901035s

[36] Stojić, G., Sremac, S., \& Vasiljković, I. (2018). A fuzzy model for determining the justifiability of investing in a road freight vehicle fleet. Operational Research in Engineering Sciences: Theory and Applications, 1(1), 62-75. https://doi.org/10.31181/oresta19012010162s

[37] Ciavarella, M., Carbone, G., \& Vinogradov, V. (2018). A critical assessment of Kassapoglou's statistical model for composites fatigue. Facta Universitatis, Series: Mechanical Engineering, 16(2), 115-126. https://doi.org/10.22190/FUME180321014C

\section{Contact information:}

\section{Radojko OBRADOVIĆ, PhD}

(Corresponding author)

University of Belgrade, Faculty of Architecture,

Bulevar kralja Aleksandra 73/Il, 11000 Belgrade, Serbia

E-mail: robradovic@hotmail.com

Dragan PAMUČAR, PhD, Associate professor University of Defence, Department of logistic, Pavla Jurisica Sturma 33, 11000 Belgrade, Serbia E-mail: dpamucar@gmail.com 